\title{
Sobre a possibilidade de determinar espectros de energia de fótons contaminantes a partir da fórmula Schiff e o algoritmo de recozimento simulado generalizado On the possibility of determining the energy spectra of photon contamination from Schiff formula and generalized simulated annealing algorithm
}

\author{
Jorge H. Wilches Visbal ${ }^{1} \odot$, Alessandro M. Da Costa ${ }^{2} \odot$ \\ ${ }^{1}$ Facultad de Ciencias de la Salud, Universidad del Magdalena, Santa Marta DTCH, Colombia \\ ${ }^{2}$ Faculdade de Filosofía, Ciências e Letras de Ribeirão Preto, Universidade de São Paulo, Ribeirão Preto, Brasil
}

\begin{abstract}
Resumo
Feixes de elétrons clínicos são compostos por uma mistura de elétrons e fótons de freamento. O espectro de energia de elétrons clínicos entende-se como a justaposição de duas componentes: componente eletrônica e componente fotônica. A componente fotônica ou espectro de fótons contaminantes pode ser obtido através de três vias: 1) Modelamento da fonte primaria; 2) Medição direta; 3) Reconstrução inversa. Neste artigo propõe-se um método de reconstrução inversa do espectro de energia dos fótons contaminantes baseado na fórmula Schiff e o algoritmo de recozimento simulado generalizado.
\end{abstract}

Palavras-chave: dose; fótons contaminantes; Schiff; recozimento simulado generalizado; Matlab; PENELOPE.

\section{Abstract}

Clinical electron beams are composed of a mixture of pure electrons and bremsstrahlung photons. The energy spectrum of clinical electron beam is composed by two components: electronic and photonic components. Photonic component named energy spectrum of photon beam can be obtained through three ways: 1) Modelling source; 2) Direct measurement and 3) Reconstruction. In this article it's proposed an inverse reconstruction method of the energy spectra of photon contamination based on Schiff formula and generalized simulated annealing algorithm.by a semicolon. References should be numbered and quoted according to the order of appearance in the article.

Keywords: dose; photon contamination; Schiff; generalized simulated annealing; Matlab; PENELOPE.

\section{Introdução}

Feixes de elétrons clínicos são compostos por uma mistura de elétrons puros e fótons de freamento (bremsstrahlung) produzidos nas estruturas internas do cabeçote do acelerador, no ar e dentro do fantoma de água. O conhecimento acurado dessas duas componentes de bremsstrahlung é importante tanto para o cálculo acurado da dose quanto para o planejamento do tratamento ${ }^{1}$.

A componente da dose de bremsstrahlung gerada no cabeçote do acelerador, conhecida como dose dos fótons contaminantes é predominante em feixes de elétrons com folhas espalhadoras, enquanto a dose de bremsstrahlung gerada no fantoma domina em feixes de elétrons de varredura ${ }^{1}$.

Em aceleradores lineares (feixes de elétrons com folhas espalhadoras), a contribuição dos fótons de freamento gerada no fantoma pode ser omitida para feixes com energia nominal menor do que $18 \mathrm{MeV}^{2}$. Contudo, na medida em que a energia nominal do feixe e o número atómico dos materiais do fantoma aumentam, essa contribuição não pode continuar a ser ignorada ${ }^{2,3}$.
A contribuição total dos fótons de freamento aparece como uma cauda tênue que diminui lentamente com a profundidade além do alcance máximo dos elétrons ${ }^{3}$ na curva da porcentagem de dose em profundidade (PDP) dos elétrons clínicos.

A curva da dose dos fótons contaminantes de feixes de elétrons clínicos possui uma forma similar à dose dos fótons de raios- $x$ de megavoltagem: região de build-up próxima da superfície; profundidade de máxima dose deslocada em profundidade e região de queda exponencial ${ }^{2-4}$.

O espectro de energia de elétrons clínicos pode ser entendido como a justaposição de duas componentes puras: componente eletrônica e componente fotônica. O espectro de energia de elétrons clínicos é útil para o cálculo acurado da dose. Métodos matemáticos para a derivação do espectro de elétrons têm sido reportados ${ }^{5,6}$.

Consequentemente, em um espectro de energia de elétrons, além do espectro dos elétrons puros, existe um espectro dos fótons contaminantes. Em artigos anteriores ${ }^{7,8}$, descrevemos como o espectro dos elétrons puros pode ser obtido, neste artigo o foco é determinar o espectro dos fótons contaminantes. 
Existem, ao menos, três abordagens pelas quais o espectro de energia dos fótons contaminantes poderia ser derivado 9 :

1) Modelamento da fonte de radiação.

2) Medição direta.

3) Reconstrução inversa.

Modelamento da fonte de radiação: exige o conhecimento da estrutura geométrica do cabeçote $\mathrm{e}$ de algum método Monte Carlo de simulação da radiação, como por exemplo PENELOPE, GAMOS ou FLUKA.

Medição direta: consiste na medição experimental da distribuição energética de fótons que atingem a superfície do fantoma usando um espectrómetro de espalhamento Compton.

Reconstrução inversa: extrai o espectro dos fótons contaminantes da dose dos fótons contaminantes e via a equação integral de Fredholm ou por meio do método analítico da fórmula aprimorada de Schiff.

Previamente, alguns autores têm realizado esforços para decifrar o espectro de energia de fótons de megavoltagem e fótons contaminantes ${ }^{2,9-11}$.

Deng et al (2001), reconstruíram o espectro de energia de fótons contaminantes por meio do algoritmo "random creep" auxiliado por resultados provenientes de simulações Monte Carlo.

Juste et al (2013), derivaram o espectro de energia de fótons de um acelerador linear se apoiando em um método de dispersão analítica.

$\mathrm{Li}$ et al (2011) obtiveram espectros de fótons de raios- $X$ de alta energia se baseando em modelos matemáticos (equação integral de Fredholm e Fórmula Schiff) e dados de dose em profundidade.

No presente trabalho se propõe a solução do problema inverso: reconstruir o espectro de energia dos fótons contaminantes (causa) a partir da dose dos fótons contaminantes (efeito) conhecida, em contraposição ao problema direto: determinar a dose dos fótons contaminantes (causa) a partir do espectro de energia dos fótons contaminantes (efeito) previamente conhecido. Para resolver o problema inverso foi utilizada uma metodologia exclusivamente matemática baseada no método analítico da fórmula Schiff e no algoritmo de recozimento simulado generalizado. Os resultados mostram um aceitável acordo entre a dose dos fótons contaminantes original e a dose dos fótons contaminantes obtida a partir do espectro dos fótons contaminantes reconstruídos, exceto na região próxima da superfície. Assim sendo, conclui-se que o método analítico é adequado para essa finalidade, mas precisa ser aprimorado.

\section{Materiais e Métodos}

A determinação do espectro de energia dos fótons contaminantes pressupõe o conhecimento prévio das curvas de doses contaminantes e a matriz de curvas de dose em profundidade monoenergéticas de raios$x$ de alta energia ${ }^{2,7}$. Isto se fez em várias etapas.

A primeira etapa é a elucidação da dose dos fótons contaminantes para cada energia nominal dos feixes de elétrons estudados. Em Visbal \& Da Costa (2017) detalha-se o procedimento de cálculo da dose dos fótons contaminantes a partir de curvas de percentagem de dose em profundidade (PDP) de elétrons clínicos.

$\mathrm{Na}$ segunda, as curvas de dose em profundidade dos fótons monoenergéticos são simuladas utilizando PENELOPE. A configuração geométrica da simulação consistiu de uma fonte pontual colocada no vácuo $100 \mathrm{~cm}$ acima de um fantoma semi-infinito de água, com apertura angular de $2,86^{\circ}$, desenhando um feixe piramidal de tamanho de campo $10 \times 10 \mathrm{~cm}^{2}$. $\mathrm{O}$ número total de feixes monoenergéticos variou de 0,125 MeV a $18 \mathrm{MeV}$ em intervalos de 0,125 MeV. A dose dos fótons monoenergéticos foi registrada a cada $0,2 \mathrm{~cm}$ de profundidade (largura do pixel) até uma profundidade máxima de $15 \mathrm{~cm}$.

A terceira etapa está relacionada com o uso da fórmula analítica de Schiff ${ }^{11-13}$ dada pela Equação (1):

$$
\begin{gathered}
\varphi(E)=\frac{e^{-\mu(E) t}}{E}\left[\frac{T-E}{T}(\ln (\eta)-1)\right. \\
\left.+\left(\frac{E}{T}\right)^{2}\left(\ln (\eta)-\frac{1}{2}\right)\right] . \\
\eta=\left(\frac{0.511 E}{2 T(T-E)}\right)^{2}+\left(\frac{Z}{111}\right)^{2} .
\end{gathered}
$$

A variável $Z$ é o número atómico efetivo dos materiais atenuantes, $T$ é a energia total dos elétrons incidentes no alvo em MeV, $t$ é a espessura hipotética do alvo em $\mathrm{cm}$ e $\mu(E)$ é o coeficiente de atenuação linear dos materiais do alvo em $\mathrm{cm}^{-1}$.

A fim de considerar as diversas formas geométricas dos sistemas de filtros dos aceleradores lineares, Deng et al (2001) propuseram a introdução da função log-normal,

$$
f(E)=c e^{-\left(\ln \left(E / E_{p}\right) / a\right)^{2} / 2},
$$

em que, $E_{p}$ é a energia pico, $a$, a largura à meia altura do pico e, $c$, uma constante de normalização.

Li et al (2011) introduziram a Eq. (2) na Eq. (1), por meio do produto $f(E) . \varphi(E)$,

$$
\begin{gathered}
\psi(E)=\frac{c e^{-\mu(E) t-\left(\ln \left(E / E_{p}\right) / a\right)^{2} / 2}}{E}\left[\frac{T-E}{T}(\ln (\eta)-1)\right. \\
\left.+\left(\frac{E}{T}\right)^{2}\left(\ln (\eta)-\frac{1}{2}\right)\right]
\end{gathered}
$$

para assim obter a que eles denominaram, Fórmula Aprimorada de Schiff.

A Eq. (3) é definida por apenas por seis parâmetros: $T, Z, t, a, c$ e $E_{p}$.

A quarta etapa consiste na minimização da norma residual,

$$
\min \left\{\left\|D(x, E) \psi(E)-D_{f c}(x)\right\|^{2}\right\},
$$

onde $D(x, E)$ é a matriz de curvas de dose de fótons monoenergéticos e $D_{f c}(x)$ a dose dos fótons contaminantes de um feixe de elétrons clínicos de energia nominal $E$ registrada no vetor profundidade $x$. 
A minimização da Eq. (4) se faz por meio da função GSA de recozimento simulado generalizado $\left(q_{v}=\right.$ 2,62, $q_{a}=-5,1000$ iterações). $O$ algoritmo de recozimento simulado generalizado tem-se explicado em um outro artigo nosso ${ }^{14}$.

Por simplicidade, tomou-se, $c=1$. O número atómico $Z$ é encontrado pela fórmula de Murty $(1965)^{15}$, supondo que os materiais degradantes formam um composto de rênio-tungstênio.

Os dados de entrada ao não terem sido simulados nem obtidos experimentalmente, foram aproximados como sendo $T=E, a=F W H M$ e $t=$ rand. FWHM é a largura à meia altura do feixe de elétrons clínicos, obtida pela expressão geral para 0 alcance terapêutico de um feixe degradado ${ }^{16}$ (Eq. (13) em Brahme \& Svensson (1979)); rand é um número aleatório entre 0 e $1^{17}$ e $E_{p}$, a energia pico do feixe de elétrons ${ }^{18}$.

Os valores dos quatro parâmetros foram restritos nas faixas a seguir:

$$
\text { Restrições }\left\{\begin{array}{c}
0.1 \leq T \leq E_{c} \\
0.01 \leq t \leq 100 \\
0.1 \leq E_{p} \leq E_{c} \\
0.01 \leq a \leq 100
\end{array},\right.
$$

em que $E_{c}$ é a energia de corte do feixe de elétrons clínico.

A energia de corte foi calculada através da equação empírica,

$$
E_{c}=2.2 R_{p}+E / 5 .
$$

$R_{p}$ é o alcance prático localizado na profundidade do $10 \%$ na curva de PDP dos elétrons clínicos de energia nominal $E^{19}$

O número de eventos simulados foi $2 \times 10^{8}$. La incerteza estadística (3 desvios padrões) foi menor do que $3 \%$ na profundidade do alcance máximo da PDP dos elétrons clínicos. A incerteza vem dada pelo mesmo PENELOPE.

\section{Resultados}

Na Figura 1 são mostrados os espectros de energia dos fótons contaminantes reconstruídos a partir da minimização da Eq. (4).

A comparação entre a dose dos fótons contaminantes reconstruída (obtida em PENELOPE a partir do espectro dos fótons contaminantes reconstruído) e a original (calculada por meio do método descrevido em Visbal \& Da Costa (2017)), para feixes de elétrons de energia nominal $6,9,12 \mathrm{e}$ $15 \mathrm{MeV}$ é apresentada na Figura 2.
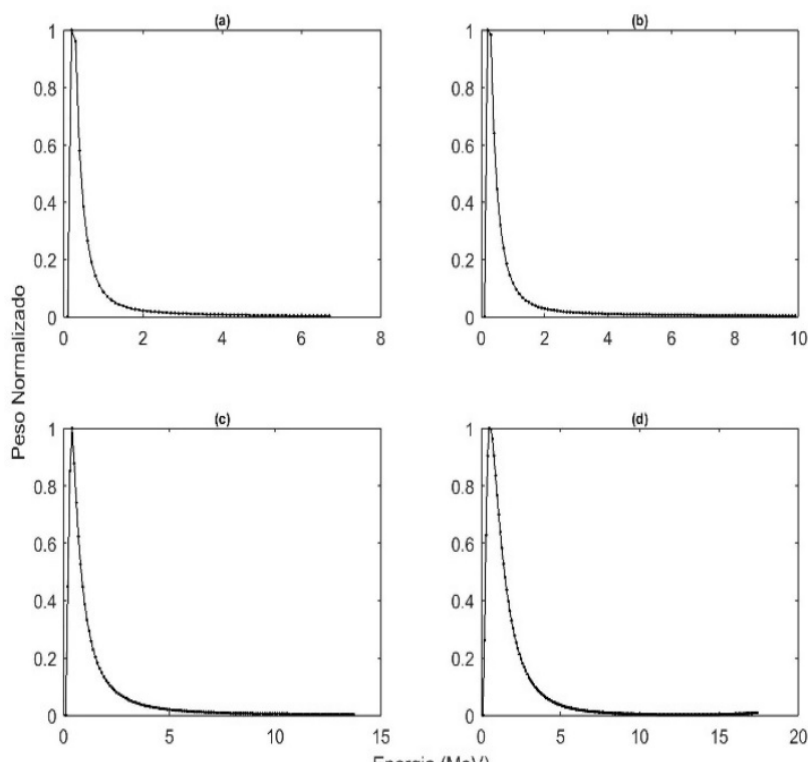

Figura 1. Espectros de energia dos fótons contaminantes reconstruidos para feixes de elétrons clínicos de energia nominal de (a) $6 \mathrm{MeV}$, (b) $9 \mathrm{MeV}$, (c) $12 \mathrm{MeV}$ e (d) $15 \mathrm{MeV}$.
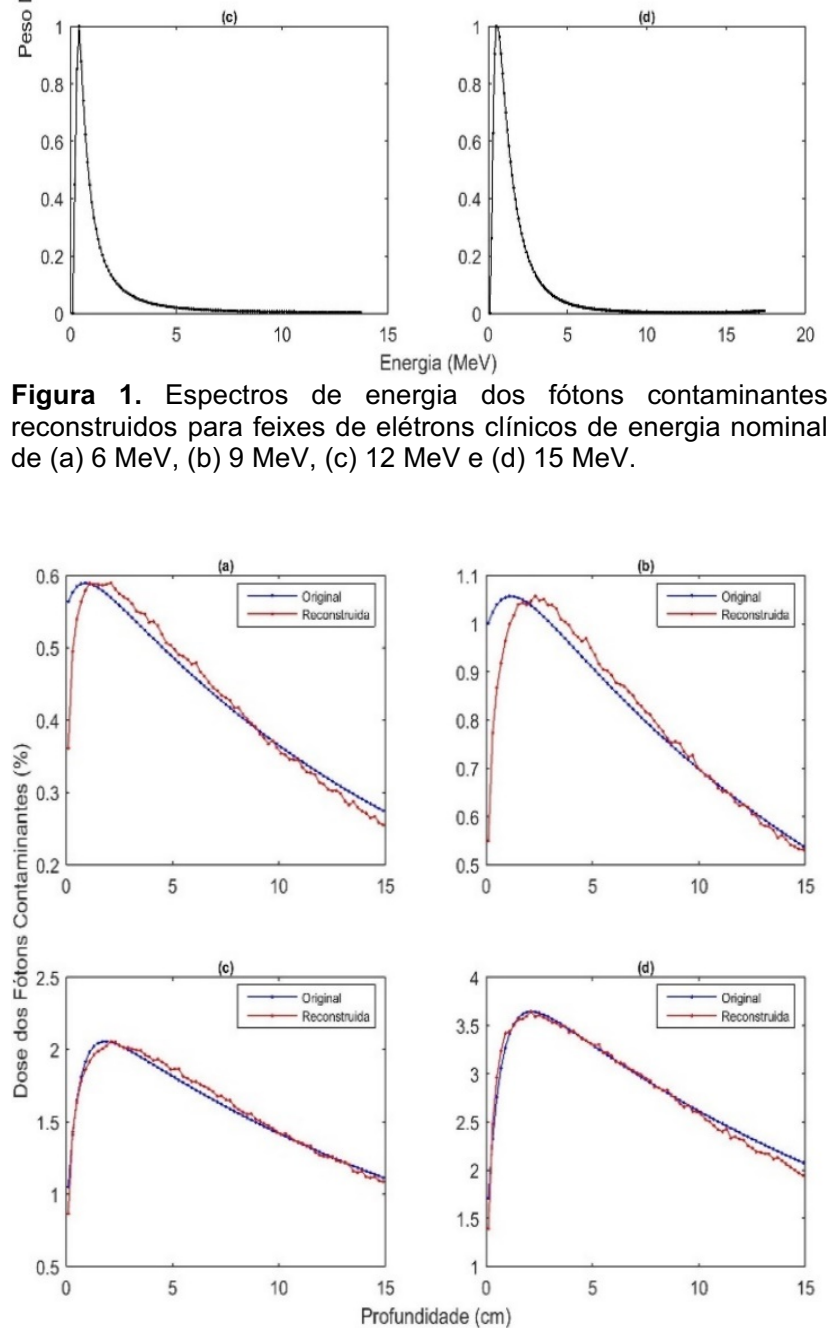

Figura 2. Comparação entre a dose dos fótons contaminantes reconstruída e original, relativa ao máximo da dose clínica (elétrons mais fótons), para feixes de elétrons clínicos de energia nominal de (a) $6 \mathrm{MeV}$, (b) $9 \mathrm{MeV}$, (c) $12 \mathrm{MeV}$ e (d) $15 \mathrm{MeV}$.

Entretanto, na Figura 3, ilustra-se a comparação entre a PDP dos elétrons puros somada com a dose dos fótons contaminantes reconstruída (curva vermelha) e somada com a dose dos fótons contaminantes original (curva azul). 


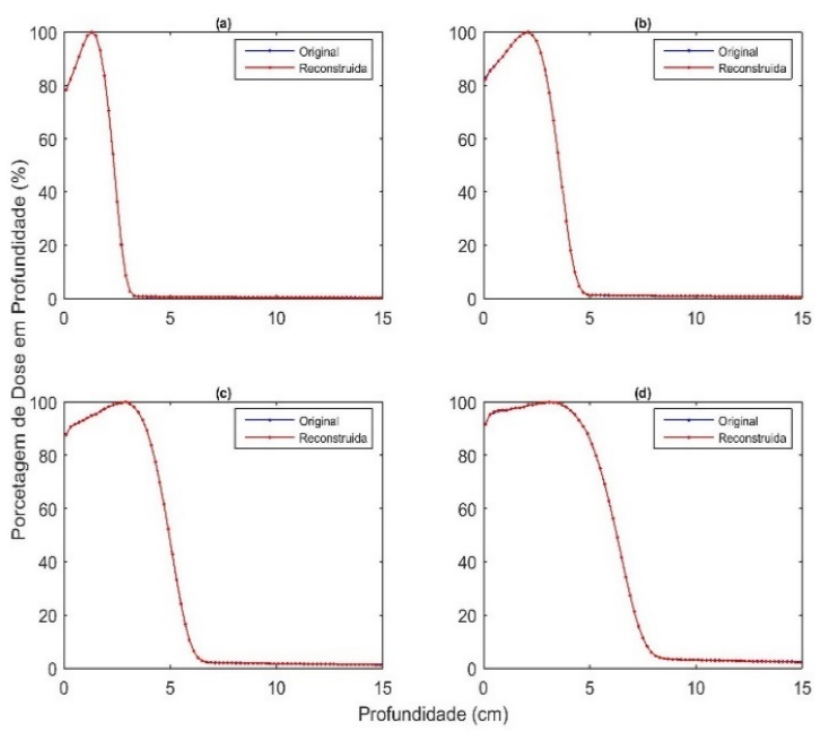

Figura 3. Comparação entre as PDP dos elétrons clinicos usando a dose dos contaminantes, original e reconstruida, para feixes de energia nominal de (a) $6 \mathrm{MeV}$, (b) $9 \mathrm{MeV}$, (c) $12 \mathrm{MeV}$ e (d) $15 \mathrm{MeV}$.

Na tabela 1 observam-se os erros médios absolutos (EMA) entre a dose dos fótons contaminantes original e reconstruída e entre as PDP dos elétrons puros com a dose dos contaminantes original e reconstruída, para os feixes de elétrons de energia nominal de 6,9 , 12 e $15 \mathrm{MeV}$.

Tabela 1. Erro médio absoluto da comparação entre as doses de fótons contaminantes original e reconstruidas (CDfco-r) e entre a PDP dos elétrons com as doses de fótons contaminantes original e reconstruidas (CPDPo-r), pp: pontos percentuais.

\begin{tabular}{ccc}
$\begin{array}{c}\text { Energia Nominal do Feixe } \\
\text { de Elétrons (MeV) }\end{array}$ & Erro Médio Absoluto (EMA) \\
\cline { 2 - 3 }$(\mathbf{p p})$ \\
$\mathbf{6}$ & CDfco-r & CPDPo-r \\
$\mathbf{9}$ & 0,016 & 0,016 \\
$\mathbf{1 2}$ & 0,034 & 0,031 \\
$\mathbf{1 5}$ & 0,032 & 0,031 \\
& 0,052 & 0,054 \\
\hline
\end{tabular}

\section{Discussão}

Como visto na Fig. 1, o espectro de energia dos fótons contaminantes é composto por um pico em baixa energia seguido por uma curva descendente para altas energias de baixa ponderação espectral.

A energia de pico nos espectros de fótons contaminantes tende a subir conforme aumenta a energia nominal do feixe de elétrons, sendo localizada em 0,1 MeV, 0,1 MeV, 0,4 MeV e 0,5 MeV para os feixes de $6,9,12$ e $15 \mathrm{MeV}$ de energia nominal respectivamente. Isto contrasta com os resultados de Deng et al (2001) em que a energia do pico não mudou com a energia nominal do feixe de elétrons.

Na Fig. 2, as maiores discrepâncias entre as curvas de dose dos contaminantes apresentaram-se em feixes de energia nominal mais baixa, particularmente perto da superfície, nos quais a incidência da dose dos fótons não é definitiva ${ }^{2,7}$.

Apesar destas diferenças entre as doses dos contaminantes, na Fig. 2, os erros médios absolutos mostrados na Tab.1, não se traduzem em desacordos clinicamente inaceitáveis nas PDP dos elétrons posto que, como claramente nota-se na Fig. 3 , qualquer diferença entre elas é imperceptível.

Muito embora dos nossos resultados não serem ótimos quanto os de Deng et al (2001), vale frisar que nós utilizamos um método puramente matemático, enquanto Deng et al (2001) usufruíram da Simulação Monte Carlo para conhecer, antecipadamente, os valores de $a$ e $E_{p}$.

Afinar o delta de energia talvez poderia conduzir a um melhor ajuste entre as curvas da Fig. 2, que significaria um espectro dos fótons contaminantes mais próximo do real. Assim mesmo, o conhecimento apropriado da faixa de variação dos parâmetros poderia conduzir a uma situação mais realista.

Por outra parte, observou-se que mudando os valores de $q_{a}$ e qv para -5 e 2,7, respectivamente, não se encontraram melhoras apreciáveis no espectro reconstruído. Além disso, quando se adicionou uma função de regularização à Eq. (4), ao não se ter um valor inicial do espectro dos fótons contaminantes, não houve uma melhoria apreciável. Inclusive, os resultados pioraram para valores altos do parâmetro de regularização.

Acreditamos que o uso de um método auxiliar para estabelecer um valor inicial do espectro de fótons contaminantes poderia melhorar os resultados, uma vez que aprimoraria a funcionalidade da função de regularização. Também, é possível que o aumento do número de iterações possa resultar em melhores resultados embora à custa do incremento no tempo de cálculo.

A reconstrução do espectro de energia dos fótons contaminantes faz parte de um processo escalonado cujo intuito é a derivação do espectro de energia dos elétrons clínicos. Com efeito, o espectro dos fótons contaminantes constitui a última etapa que começa com a reconstrução do espectro puro dos elétrons, continuando com a extração da dose dos fótons contaminantes. Para uma melhor compreensão, recomendamos revisar os nossos anteriores trabalhos Visbal \& Da Costa (2016), Visbal \& Da Costa (2017) e Visbal \& Da Costa (2019).

O software utilizado para o processamento dos dados foi: MATLAB® R2015a, Windows 7, SP1. CPU: 2,3GHz e RAM: $4 \mathrm{~Gb}$.

\section{Conclusões}

O método de reconstrução de espectro de fótons contaminantes, baseado na fórmula aprimorada de Schiff e o algoritmo de recozimento simulado, demonstrou resultados aceitáveis do ponto de vista prático.

Informação a priori acerca dos parâmetros de entrada, dentre eles o espectro inicial, poderia melhorar os resultados, sobretudo na região próxima da superfície, para feixes de energia mais baixa.

A despeito de terem se mudados os parâmetros de entrada de GSA ( $q_{a}$ e $q_{v}$ ), os resultados não foram aprimorados significativamente. O uso de uma função de regularização também não deu certo, provavelmente pela falta do espectro inicial de referência. 


\section{Agradecimentos}

Os autores agradecem o apoio da Comissão de Aperfeiçoamento de Pessoal do Nível Superior (CAPES) e à Fundação de Amparo e Pesquisa do Estado de São Paulo (FAPESP).

\section{Referências}

1. Zhu, Timothy C., Indra J. Das, and Bengt E. Bjärngard. "Characteristics of bremsstrahlung in electron beams." Medical physics 28.7 (2001): 1352-1358.

2. Deng, Jun, et al. "Derivation of electron and photon energy spectra from electron beam central axis depth dose curves." Physics in medicine and biology 46.5 (2001): 1429.

3. Rustgi, Surendra N., and James E. Rodgers. "Analysis of the bremsstrahlung component in 6-18 MeV electron beams." Medical physics 14.5 (1987): 884-888.

4. Sorcini, B. B., S. Hyödynmaa, and A. Brahme. "The role of phantom and treatment head generated bremsstrahlung in high-energy electron beam dosimetry." Physics in medicine and biology 41.12 (1996): 2657.

5. Gui, Li, et al. "Realization and comparison of severa regression algorithms for electron energy spectrum reconstruction." Chinese Physics Letters 25.7 (2008): 2710.

6. $\mathrm{Li}, \mathrm{Gui}$, et al. "Electron spectrum reconstruction as nonlinear programming model using micro-adjusting algorithm." 7th Asian-Pacific Conference on Medical and Biological Engineering. Springer, Berlin, Heidelberg, (2008).

7. Visbal, Jorge Homero Wilches, and Alessandro Martins Da Costa. "Determinação da Dose dos Fótons Contaminantes de Feixes de Elétrons Clínicos usando o Método de Recozimento Simulado Generalizado." Revista Brasileira de Física Médica 11.2 (2017): 2-6.

8. Visbal, Jorge Homero Wilches, and Alessandro Martins Da Costa. "Determinação de Espectros de Energia de Elétrons Clínicos a partir de Curvas de Porcentagem de Dose em Profundidade (PDP) utilizando o Método de Recozimento Simulado Clássico." Revista Brasileira de Física Médica 10.3 (2016): 7-10.

9. $\mathrm{Li}, \mathrm{Gui}$, et al. "Photon energy spectrum reconstruction based on Monte Carlo and measured percentage depth dose in accurate radiotherapy." Progress in Nuclear Science and Technology 2 (2011): pp-160.

10. Nisbet A, Weatherburn H, Fenwick JD, McVey G. Spectral reconstruction of clinical megavoltage photon beams and the implications of spectral determination on the dosimetry of such beams. Physics in Medicine \& Biology. 1998 Jun;43(6):1507.

11. Menin $\mathrm{OH}$, Martinez AS, Costa AM. Reconstruction of bremsstrahlung spectra from attenuation data using generalized simulated annealing. Applied Radiation and Isotopes. 2016 May 1;111:80-5.

12. Juste, B., Miró, R., Verdú, G., \& Santos, “A. Linac energy spectrum determination using the Schiff Bremsstrahlung parametric version". International Nuclear Atlantic Conference - INAC (2013).

13. Desobry, G. E., and A. L. Boyer. "Bremsstrahlung review: an analysis of the Schiff spectrum." Medical physics 18.3 (1991): 497-505.

14. Visbal JW, Costa AM. Inverse reconstruction of energy spectra of clinical electron beams using the generalized simulated annealing method. Radiation Physics and Chemistry. 2019 Sep 1;162:31-8.

15. Murty, R. C. "Effective atomic numbers of heterogeneous materials." Nature 207.4995 (1965): 398.

16. Brahme, A., and H. Svensson. "Radiation beam characteristics of a $22 \mathrm{MeV}$ microtron." Acta radiologica: oncology, radiation, physics, biology 18.3 (1979): 244-272.

17. Uniformly distributed random numbers. MathWorks. 2018. Disponível em : https://www.mathworks.com/help/matlab/ref/rand.html.

18. Deasy, J. O., P. R. Almond, and M. T. McEllistrem. "The spectral dependence of electron central-axis depth-dose curves." Medical physics 21.9 (1994): 1369-1376.

19. Gerbi, Bruce J., et al. "Recommendations for clinical electron beam dosimetry: supplement to the recommendations of Task Group 25." Medical physics 36.7 (2009): 3239-3279.

\section{Contato:}

Autor Correspondente:

Jorge Homero Wilches Visbal

Facultad de Ciencias de la Salud, Universidad del Magdalena

Calle 32 No. 22 - 08, Sector San Pedro Alejandrino, Santa Marta DTCH, Colômbia.

E-mail jhwilchev@gmail.com

Alessandro M. Da Costa

Faculdade de Filosofia, Ciências e Letras de Ribeirão Preto, Universidade de São Paulo, Ribeirão Preto, Brasil

E-mail amcosta@usp.br 